\title{
Endoscopic Treatment of Jejunal Heterotopic Gastric Mucosa that Caused Recurrent Intussusception
}

\author{
Ke Ryun Ahn', Ja Seol Koo', Hwan II Kim¹, Ji Hye Kim¹, Jee Hyun Lee'², Seung Young Kim¹, Sung Woo Jung ${ }^{1}$ and Sang Woo Lee \\ Department of ${ }^{1}$ Internal Medicine, ${ }^{2}$ Pediatrics, Korea University College of Medicine, Seoul, Korea
}

Heterotopic gastric mucosa (HGM) is a rare anomaly in the small bowel and may be the cause of intussusception when it gets a lead point in the jejunum. All cases of intussusception due to intestinal HGM have been treated with surgical resection. A 5-year-old girl presented with chief complaints of vomiting and abdominal pain for 2 weeks. A computed tomography scan of the abdomen showed intussusception at the proximal jejunal loops. Three air reductions and one saline reduction were attempted without success. She continued to be symptomatic, and endoscopic evaluation was performed. Enteroscopy revealed some variable-sized polypoid mucosal lesions with erosions on the proximal jejunum. Endoscopic mucosal resection was performed using a snare. The resected tissues histologically showed a hyperplastic polyp arising from the HGM. Her symptoms did not recur within 1 year after the treatment. Our case showed that enteroscopy could be useful for the diagnosis and management of jejunal intussusception caused by HGM. Clin Endosc 2017;50:605-608

Key Words: Heterotopic gastric mucosa; Recurrent jejunal intussusception; Endoscopic mucosal resection; Jejunal polyp

\section{INTRODUCTION}

Intussusception is a process where the bowel invaginates into the neighboring bowel lumen. It commonly causes gastrointestinal (GI) obstruction in children aged between 2 and 12 months, and is a frequent life-threatening acute abdominal emergency. ${ }^{1}$

The incidence of recurrent intussusception ranges from $2 \%$ to $20 \%$. Multiple recurrences of intussusception are associated with a pathological lead point. ${ }^{2,3}$ A lead point is a lesion or variation in the intestines that is trapped by peristalsis and dragged into a distal segment of the intestines, causing intussusception. A vascular malformation, polyp, tumor, hematoma, or Meckel's diverticulum can be a lead point of intussus-

Received: January 31, 2017 Revised: August 8, 2017

Accepted: August 21, 2017

Correspondence: Ja Seol Koo

Department of Internal Medicine, Korea University Ansan Hospital, Korea University College of Medicine, 123 Jeokgeum-ro, Danwon-gu, Ansan 15355, Korea

Tel: +82-31-412-4853, Fax: +82-31-412-5582, E-mail: jskoo@korea.ac.kr

(c) This is an Open Access article distributed under the terms of the Creative Commons Attribution Non-Commercial License (http://creativecommons.org/ licenses/by-nc/3.0) which permits unrestricted non-commercial use, distribution, and reproduction in any medium, provided the original work is properly cited. ception. $^{4}$

Heterotopic gastric mucosa (HGM) is a rare anomaly in the small bowel and originates from the vitello-intestinal tract. Although HGM occurs rarely in the jejunum, it may cause intussusception as a lead point in the jejunum. ${ }^{5}$

Surgical treatment is usually recommended for recurrent intussusception to reduce the intussusception and resect the lead point. ${ }^{6}$ All reported cases of jejunal intussusception due to jejunal HGM were treated with surgical resection of the lesion. ${ }^{5}$

Herein, we report a case of jejunal HGM that caused recurrent intussusception and treated successfully using endoscopic resection.

\section{CASE REPORT}

A 5-year-old girl was admitted to the emergency department owing to vomiting and abdominal pain for 2 weeks. She was born at 40 weeks (full term), weighing $2.4 \mathrm{~kg}$, and received full vaccination up-to-date. Her medical and family histories were unremarkable. At admission, her height and weight were $116 \mathrm{~cm}$ and $20 \mathrm{~kg}$, respectively. On physical ex- 
amination, she had tenderness and rebound tenderness at the epigastric area. However, no mass was palpated on her abdomen. The laboratory findings revealed the following values: hemoglobin (Hb), $14.3 \mathrm{~g} / \mathrm{dL}$; hematocrit (Hct), 41.4\%; white blood cells (WBC), 19,680/ $\mu \mathrm{L}$; and platelets, $464,000 / \mathrm{mL}$. The C-reactive protein (CRP) level was slightly increased $(0.33 \mathrm{mg} /$ dL). Her stool $\mathrm{Hb}$ level was $201 \mathrm{ng} / \mathrm{mL}$, although hematochezia or melena was not detected on rectal examination.

On abdominal ultrasonography (US), an intussuscepted inner segment was detected on the edematous outer bowel, at the left upper quadrant of the abdomen (Fig. 1). Abdominal computed tomography (CT) revealed an intussusception at the proximal jejunal loops without a lead point (Fig. 2). Three air reductions and one saline reduction were attempted. However, her symptoms recurred, and repeated US still detected jejunal intussusception. On laparoscopic examination for jejunal reduction and investigation of a lead point of intussusception, no lead point was detected. She still complained of intermittent abdominal pain 1 month after the laparoscopic

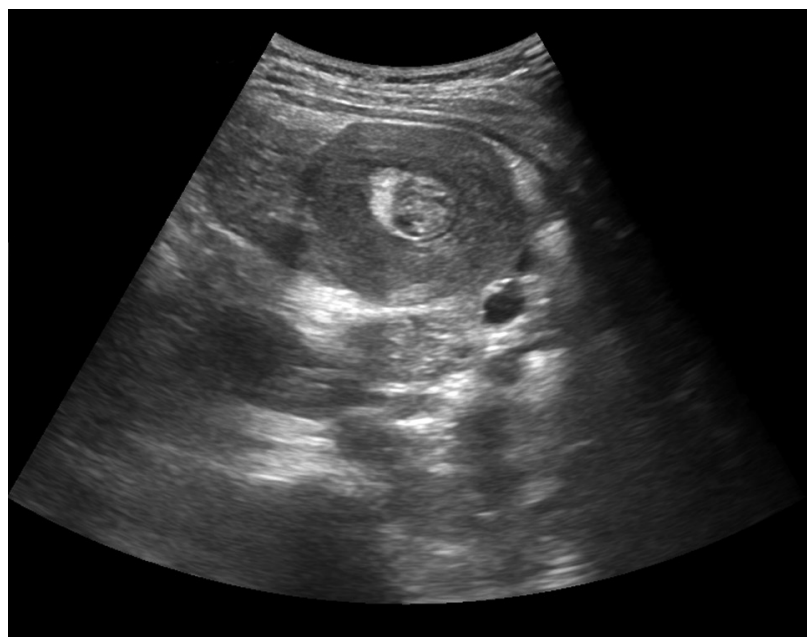

Fig. 1. Abdominal ultrasonography shows an intussuscepted inner segment and edematous outer bowel in the left upper quadrant. examination. Capsule endoscopy was not performed owing to the risk of retention. Therefore, enteroscopy using a gastroscope (GIF-Q260; Olympus, Tokyo, Japan) was performed under fluoroscopic guidance and sedation. Enteroscopy revealed a few variable-sized polypoid lesions with erosions on the proximal jejunum. The largest lesion nearly obstructed the jejunal lumen (Fig. 3). On histopathological examination of the tissues obtained via endoscopic biopsy, chronic inflammation was found without specific findings for diagnosis. After obtaining the histopathological result, we repeated the enteroscopy to remove the jejunal lesions. In the second enteroscopy, the total procedure time was 55 minutes, and moderate

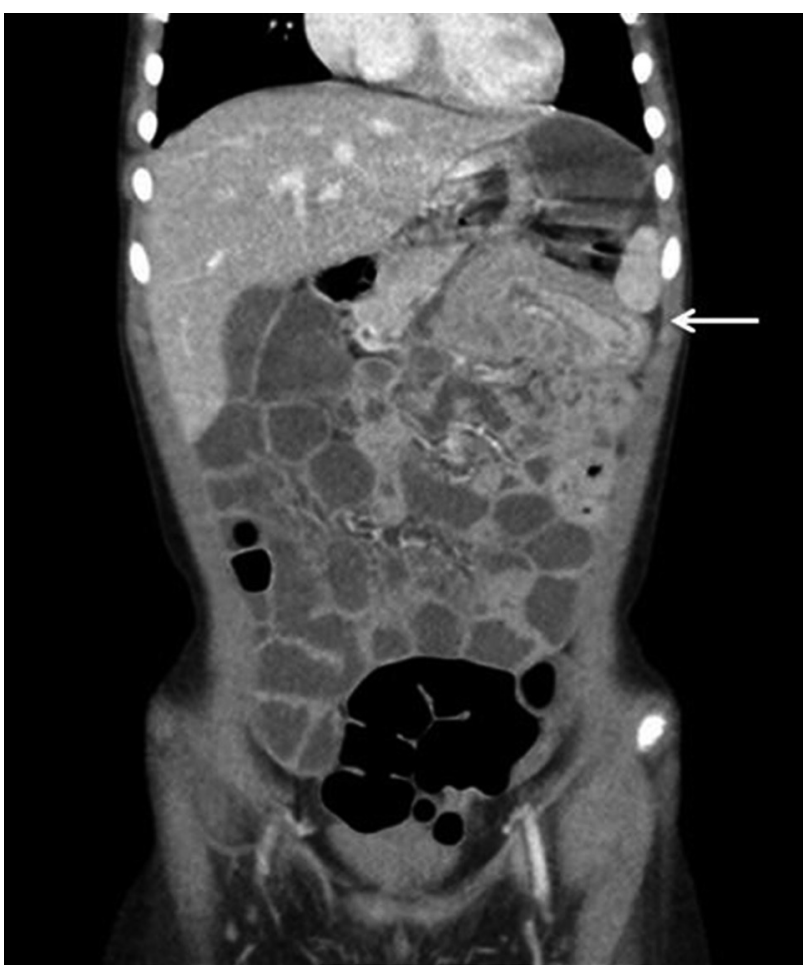

Fig. 2. Abdominal computed tomography further localizes the intussusception in the proximal jejunal loops (arrow).
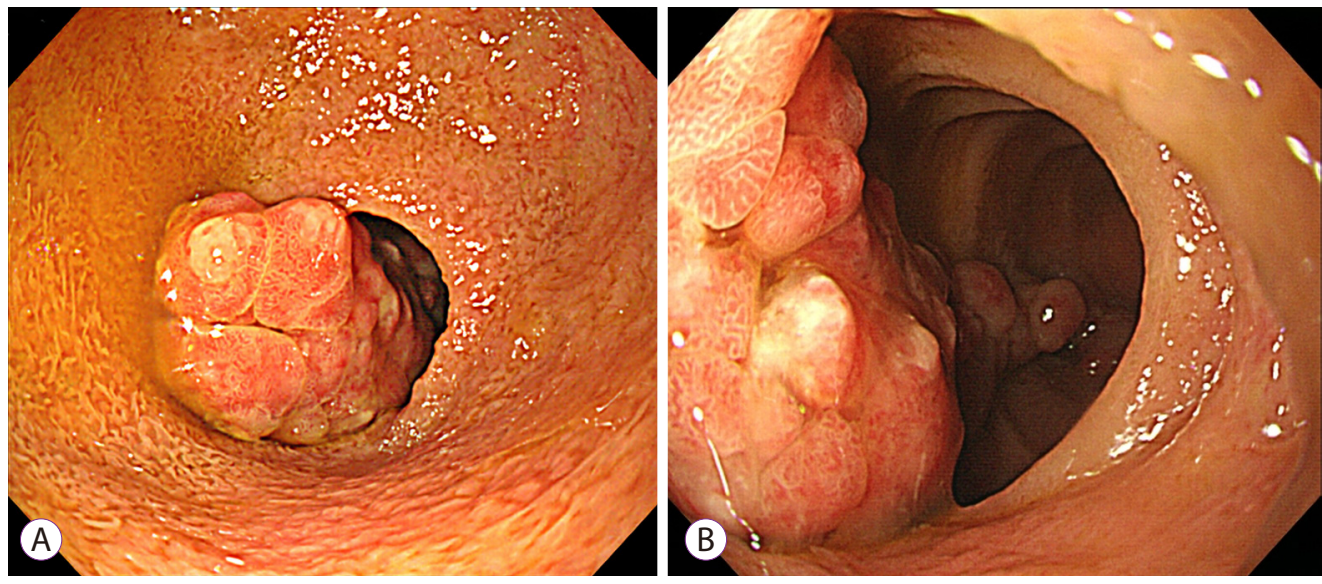

Fig. 3.Endoscopic images of the polypoid lesions in the proximal jejunum. (A) The largest polyp almost completely fills the jejunal lumen. (B) Variable-sized polypoid mucosal lesions with erosions. 


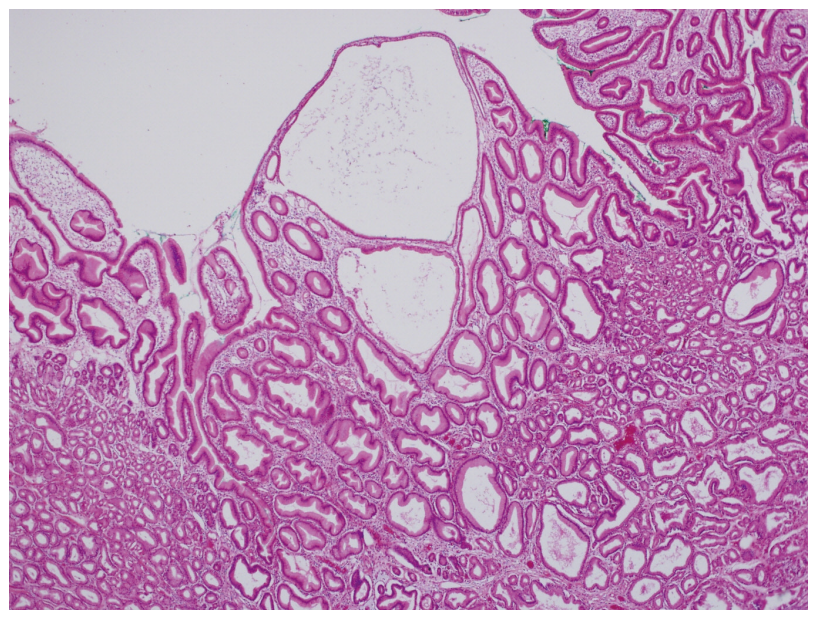

Fig. 4. Histopathologic evaluation shows a foveolar epithelium and cystic change in the hyperplastic polyps arising from the gastric mucosa (Hematoxylin \& eosin staining, $\times 20$ ).

sedation was achieved using midazolam and propofol. During the procedure, a total of $6 \mathrm{mg}$ of midazolam and $170 \mathrm{mg}$ of propofol were used. After the enteroscopy, she recovered from the sedation without any complications.

Histopathological evaluation of the resected tissues showed a foveolar epithelium with a cystic change (Fig. 4). On immunohistochemical staining, the polyps expressed MUC4, which is present in the normal gastric mucosa; MUC5AC, which is expressed in the foveolar cells of the body and the antrum; MUC6, which is expressed in the gastric antral and pyloric glands; and the focally expressed MUC2 marker. However, it showed a negative expression for MUC1. Finally, the polyps were diagnosed as hyperplastic polyps arising from the HGM. Her symptoms did not recur within 1 year after the endoscopic treatment.

\section{DISCUSSION}

Recurrent intussusceptions are rare, with an incidence rate of $10 \%$. However, the recurrence rate is dramatically increased with the number of previous recurrences and ranges from 5.5\% during the first recurrence to $37.5 \%$ during the third episode. ${ }^{8}$ Several diseases such as Meckel's diverticulum, polyp, tumor, hematoma, vascular malformation, or HGM can result in intestinal intussusception. ${ }^{4,5}$

HGM can occur throughout the GI tract, up to the oral cavity down to the anorectum, including the airways, umbilicus, gallbladder, biliary tree, urinary bladder, and scrotum. ${ }^{9,10}$ HGM can either be congenital or acquired. Congenital HGM may occur in patches around the esophagus, Meckel's diverticulum, and intestinal duplications or as nodular masses in the duodenum. However, congenital isolated HGM distal to the ligament of Treitz is rare.

The clinical presentation varies and depends on the size and location of the HGM. HGM can form an intraluminal mass and cause airway or intestinal obstruction. ${ }^{11,12}$ It can serve as a lead point for the development of intussusception. ${ }^{13,14}$

Intestinal mucosal ulceration with GI bleeding is a known complication of HGM. ${ }^{15}$ Intestinal perforation and fistulas to adjacent structures have also been described. ${ }^{16}$

Radiological investigations such as US, contrast radiography of the upper GI tract, and CT may reveal intussusceptions or intraluminal polypoid lesions. ${ }^{17}$ Contrast radiography of the upper GI tract and US demonstrated the obstruction and intussusception on the jejunal lesion in the present case.

Surgery is used to achieve the reduction of intussusception and to evaluate the lesion histopathologically. Surgery, including resection of the jejunal lesion and anastomosis of the healthy jejunal ends, has been considered as the treatment of choice. ${ }^{5}$ It is especially recommended for the treatment of patients with recurrent intussusception. ${ }^{6}$ In a review of reported cases of intussusception caused by HGM, all cases were managed using surgical methods such as surgical polypectomy or intestinal resection and anastomosis. ${ }^{5}$

However, after surgical reduction of the intussusception, the overall recurrence rate was $8.2 \%{ }^{2}$ In a case with recurrent ileal intussusception, an emergency surgery was performed to relieve the intussusception and resect the ileum, including the HGM, after two open reductions. ${ }^{1}$ Therefore, an endoscopy, instead of a surgical treatment, can be performed to manage the lesion in intussusceptions recurring after surgical reduction as in our case.

Enteroscopy is useful for diagnosing and treating small bowel diseases in children. Yokoyama et al. reported 46 cases of enteroscopic polypectomy in patients with hereditary polyposis syndrome who were aged 18 years and younger. ${ }^{18}$ Most procedures were successful, and only four patients had post-polypectomy bleeding and needed hemostatic therapy. ${ }^{18}$ In a previous report, enteroscopic polypectomy did not result in severe complications in children younger than 16 years who had Peutz-Jeghers or Cowden syndrome. ${ }^{19}$ Although enteroscopy in children seems to be safe, clinicians must recognize that this procedure is quite invasive and should be performed in selected patients with severe small bowel diseases. ${ }^{19220}$

Before performing endoscopy in children, anxiety relief is needed to conduct a successful examination. The presence of parents is usually essential, and premedication with benzodiazepines, for example, can be used to reduce anxiety. General anesthesia or deep sedation is usually necessary; thus, physicians should be cautious, as children are more vulnerable to respiratory complications owing to their greater lung resistance. ${ }^{20}$ 
This is the first case of recurrent jejunal intussusception due to HGM that was treated with endoscopic resection, although all previously reported cases were treated with surgical management. Therefore, physicians should consider performing enteroscopy for the diagnosis and treatment of lesions in patients with recurrent intussusception.

Conflicts of Interest

The authors have no financial conflicts of interest.

\section{REFERENCES}

1. Elemen L, Oz F, Erdogan E. Heterotopic gastric mucosa leading to recurrent intussusceptions: report of a case. Surg Today 2009;39:444-447.

2. Fecteau A, Flageole H, Nguyen LT, Laberge JM, Shaw KS, Guttman FM. Recurrent intussusception: safe use of hydrostatic enema. J Pediatr Surg 1996;31:859-861.

3. Yang CM, Hsu HY, Tsao PN, Chang MH, Lin FY. Recurrence of intussusception in childhood. Acta Paediatr Taiwan 2001;42:158-161.

4. Navarro O, Daneman A. Intussusception. Part 3: diagnosis and management of those with an identifiable or predisposing cause and those that reduce spontaneously. Pediatr Radiol 2004;34:305-312; quiz 369.

5. Boybeyi O, Karnak I, Güçer S, Orhan D, Senocak ME. Common characteristics of jejunal heterotopic gastric tissue in children: a case report with review of the literature. J Pediatr Surg 2008;43:e19-e22.

6. Hsu WL, Lee HC, Yeung CY, et al. Recurrent intussusception: when should surgical intervention be performed? Pediatr Neonatol 2012;53:300-303.

7. Reis CA, David L, Carvalho F, et al. Immunohistochemical study of the expression of MUC6 mucin and co-expression of other secreted mucins (MUC5AC and MUC2) in human gastric carcinomas. J Histochem Cy- tochem 2000;48:377-388

8. Ksia A, Mosbahi S, Brahim MB, et al. Recurrent intussusception in children and infants. Afr J Paediatr Surg 2013;10:299-301.

9. Agha FP, Ghahremani GG, Tsang TK, Victor TA. Heterotopic gastric mucosa in the duodenum: radiographic findings. AJR Am J Roentgenol 1988;150:291-294

10. Galligan ML, Ulich T, Lewin KJ. Heterotopic gastric mucosa in the jejunum causing intussusception. Arch Pathol Lab Med 1983;107:335-336.

11. Daher P, Riachy E, Zeidan S, Saad A. Upper airway obstructive symptoms because of ectopic gastric mucosa in a newborn: a case report. J Pediatr Surg 2006;41:e7-e9.

12. Lui B, Korman B. Congenital oral heterotopic gastrointestinal cyst: case report and review of the literature. J Otolaryngol Head Neck Surg 2008;37:E151-E154.

13. Erez I, Kovalivker M, Lew S, Lazar L, Motovic A. Ectopic gastric mucosa in a polyp causing ileo-ileal intussusception: a case report of a threemonth-old baby. Eur J Pediatr Surg 1991;1:118-120.

14. Bertin P. [Ileo-ileal intussusception over an islet of heterotopic gastric mucosa without Meckel's diverticulum]. Chir Pediatr 1981;22:7-11.

15. Jimenez JC, Emil S, Steinmetz B, Romansky S, Weller M. Recurrent gastrointestinal tract bleeding secondary to jejunal gastric heterotopia. J Pediatr Surg 2005;40:1654-1657.

16. Acea Nebril B, Bouso Montero M, Blanco Freire N, et al. [Heterotopic gastric mucosa in the ileum with perforated ulcer]. Gastroenterol Hepatol 1996;19:514-516.

17. Lodge JP, Brennan TG, Chapman AH. Heterotopic gastric mucosa presenting as small-bowel obstruction. Br J Radiol 1987;60:710-712.

18. Yokoyama K, Yano T, Kumagai H, et al. Double-balloon enteroscopy for pediatric patients: evaluation of safety and efficacy in 257 cases. J Pediatr Gastroenterol Nutr 2016;63:34-40.

19. Thomson M, Venkatesh K, Elmalik K, van der Veer W, Jaacobs M. Double balloon enteroscopy in children: diagnosis, treatment, and safety. World J Gastroenterol 2010;16:56-62.

20. Friedt M, Welsch S. An update on pediatric endoscopy. Eur J Med Res 2013;18:24 